\title{
Prostate cancer, ESMO 2016
}

\author{
Bobby Shayegan, MD, FRCSC
}

Associate Professor, Department of Surgery, McMaster University, Hamilton, ON, Canada

\section{Long-term safety of abiraterone acetate + prednisone in chemotherapy-naiive metastatic CRPC}

\section{Long-term results of chemohormonal therapy in metastatic prostate cancer}

The CHAARTED trial prospectively compared androgendeprivation therapy (ADT) to ADT plus docetaxel in metastatic hormone-sensitive prostate cancer. ${ }^{1}$ The 790 patients in this trial were prospectively stratified into high-volume (visceral metastases and/or four or more bone metastases with at least one outside of the vertebral column and pelvis) and low-volume disease.

Long-term results presented at ESMO 2016 showed that ADT plus docetaxel was associated with a significant improvement in median overall survival (OS) compared to ADT alone (57.6 months vs. 47.2 months [hazard ratio (HR) 0.73 ]; $p=0.0018)$. Subgroup analysis continues to show that only the high-volume disease group derived benefit from the addition of docetaxel (Fig. 1). The median OS in this group was 51.2 months for ADT + docetaxel and 34.4 months for ADT alone (HR 0.63; $\mathrm{p}<0.0001)$. In the low-volume subgroup, median OS was 63.5 months for the docetaxel arm and not reached in the ADT alone arm (HR 1.04; $p=0.86$ ). These observations were similar regardless of whether or not patients had prior local treatment. Benefits in time to clinical progression and quality of life (FACT-P) were also only seen in the subgroup with high-volume disease.

The only benefit of ADT + docetaxel compared to ADT alone among low-volume patients was observed in time to castration-resistant prostate cancer (CRPC) (prostate-specific antigen [PSA] rise or clinical progression: median 31.0 months with ADT + docetaxel, 22.7 months with ADT alone; $\mathrm{p}=0.03$ ). This data is likely going to impact decision-making in administration of docetaxel to young patients who present with limited and low-volume metastatic disease.
Abiraterone acetate with prednisone $(A A+P)$ is a first-line therapeutic option for men with metastatic CRPC (mCRPC). Safety and efficacy of this agent were demonstrated in the pivotal COU-AA-302 trial. Unlike the era of cytotoxic agents for $\mathrm{mCRPC}$, increasing numbers of patients are now being treated and remaining alive with disease for prolonged periods of time, raising concerns regarding long-term safety.

At ESMO 2016, investigators presented long-term safety data from the pivotal COU-AA-302 trial in men treated for $\geq 4$ and $<4$ years. $^{2}$ Baseline characteristics were similar in the two cohorts of $\geq 4$ and $<4$ years, with the exception of median age (67 and 71 years, respectively) and time from androgen-deprivation to treatment with AA + P (56.3 and 39.3 months, respectively). Median treatment duration was 49.7 months in the $\geq 4$-year group (41 patients) and 12.7 months for the $<4$-year group (505 patients). Rates of overall adverse events (AEs), Grade 3/4 AEs, and serious AEs were similar between the 0 - to 12 -month and 36- to 48-month treatment periods. No patients in the $\geq 4$-year group had AEs leading to therapy discontinuation and 50 patients in the $<4-$ year group discontinued treatment due AEs.

In summary, there were no new safety signals detected during long-term followup and no signs of cumulative toxicity over time. Prolonged therapy with $\mathrm{AA}+\mathrm{P}$ remains efficacious, with low toxicity and low discontinuation in those treated for prolonged periods of time.

\section{AR-V7: A less robust predictor of enzalutamide resistance in first-line treatment of $m C R P C$}

The PREMIERE trial is a multicentre, single-arm, open-label phase 2 clinical trial of enzalutamide in first-line among 98 chemotherapy-naïve patients with mCRPC. ${ }^{3}$ An analysis of the PREMIERE dataset presented at ESMO 2016 sought 


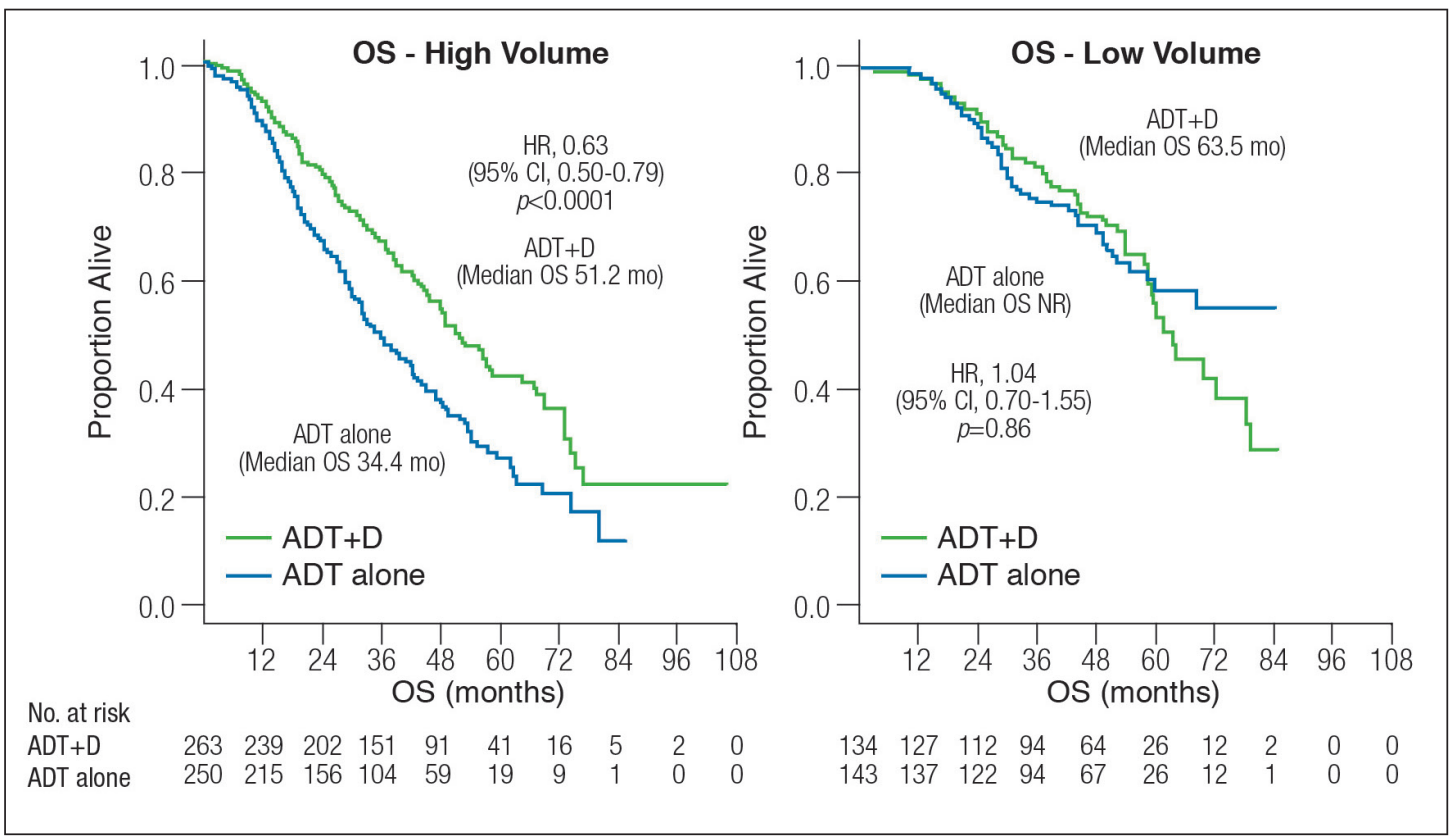

Fig. 1. Overall survival among patients with high or low burden of metastatic disease, $A D T+$ docetaxel vs. ADT alone (CHAARTED trial). ADT: androgen-deprivation therapy; D: docetaxel; Cl: confidence interval; HR: hazard ratio; OS: overall survival.

to determine the predictive value of AR-V7 expression on response to enzalutamide therapy in first-line use.

AR-V7 expression in circulating tumour cells (CTCs) was found in $24.2 \%$ (eight patients). Among these patients, $50.0 \%$ demonstrated $>50 \%$ decline in PSA when treated with enzalutamide. A further $37.5 \%$ of AR-V7 patients showed $>90 \%$ PSA response to first-line therapy.

The investigators concluded that, contrary to previous findings with enzalutamide in the second-line setting, the detection of AR-V7 expression in CTCs is not an absolute predictor for early resistance to enzalutamide in the firstline setting. However, further followup is needed to assess impact on OS.

\section{Second-line custirsen + cabazitaxel/prednisone in mCRPC}

The AFFINITY study investigated the role of the combination of custirsen (an inhibitor of clusterin, a cytoprotective protein that prevents apoptosis and confers treatment resistance) and cabazitaxel/prednisone compared to cabazitaxel/ prednisone alone in 635 patients with mCRPC who had progressed on docetaxel. ${ }^{4}$

Patients were randomized to 21-day cycles of custirsen plus cabazitaxel/prednisone or cabazitaxel/prednisone plus placebo. Treatments were continued until progression, unacceptable toxicity, or completion of 10 cycles. The coprimary endpoints were OS in the entire cohort and OS in the subset of patients with poor prognosis, defined as hav- ing two or more of: Karnofsky Performance Status $\leq 80 \%$; presence of liver metastases; hemoglobin $<12 \mathrm{~g} / \mathrm{dL}$; lactate dehydrogenase $(\mathrm{LDH}) \geq 360 \mathrm{IU} / \mathrm{L}$; or PSA $\geq 150 \mathrm{ng} / \mathrm{mL}$. Of the 635 patients in the study, 392 fulfilled the poor prognosis criteria, with approximately equal numbers in each treatment arm.

In the entire cohort, there was no significant difference detected in OS between treatment arms. Median OS was 14.2 months for the custirsen-containing arm and 13.4 months for the control arm (HR 0.946; $p=0.264)$. There was also no significant impact of adding custirsen in the subgroup with poor prognosis (HR 0.918; $\mathrm{p}=0.235$ ) (Table 1$)$.

The rationale for the study was to examine the use of inhibitors of cell-survival pathways to decrease taxane resistance in prostate cancer. Unfortunately, the results were clearly negative. Robust biologic molecular predictors are needed to improve stratification in such trials.

\section{Pembrolizumab as second-line in advanced prostate adenocarcinoma}

Pembrolizumab is an anti-PD-1 antibody blocking the interaction of PD-1 and PD-L1. KEYNOTE-028 is a nonrandomized, phase $1 \mathrm{~b}$ trial evaluating safety and efficacy of this agent in 20 advanced solid tumour cohorts. ${ }^{5}$ The prostate adenocarcinoma cohort (23 patients) forms the basis for this presentation. Key eligibility criteria included advanced prostate cancer, failure of standard therapy, measurable disease, and PD-L1 expression in $\geq 1 \%$ of tumour or 
Table 1. No difference in OS with the addition of custirsen to cabazitaxel/prednisone in mCRPC previously treated with docetaxel (AFFINITY trial)

\begin{tabular}{|c|c|c|c|}
\hline \multirow{2}{*}{ Group } & \multicolumn{2}{|c|}{ Median overall survival, months $(95 \% \mathrm{Cl})$} & \multirow{2}{*}{ p value } \\
\hline & Cabazitaxel/prednisone + custirsen & Cabazitaxel/prednisone & \\
\hline Full cohort ( $n=635)$ & $14.2(12.6-16.0)$ & $13.4(12.0-15.2)$ & 0.264 \\
\hline Poor prognosis subset $(n=392)$ & $11.1(9.3-13.3)$ & $10.9(8.3-12.4)$ & 0.235 \\
\hline
\end{tabular}

stromal cells by immunohistochemistry. Subjects received pembrolizumab at a dose of $10 \mathrm{mg} / \mathrm{kg}$ every two weeks for two years or until disease progression. The primary efficacy endpoint was objective response rate (ORR). Safety and tolerability were assessed throughout the study period and for 90 days thereafter.

Median age in the cohort was 65 years and 74\% had received two or more prior therapies for metastatic disease. Median followup was 33 weeks. Three of 23 patients experienced a Grade 3 treatment-related AE (asthenia, fatigue, and peripheral neuropathy) and one patient experienced a Grade 4 AE (increased lipase). There were no deaths or discontinuations due to AEs among the 23 patients.

The confirmed ORR was $13.0 \%$ (3/23 patients). There were an additional two patients who achieved an unconfirmed partial response (unconfirmed ORR 21.7\% [5/23 patients]). Median OS was 7.9 months, with six-month and 12-month OS rates of $73.4 \%$ and $34.0 \%$, respectively. Median progression-free survival (PFS) was 3.6 months, with six-month and 12 -month PFS rates of $39.1 \%$ and $14.7 \%$, respectively.

It is important to note that these were heavily pretreated patients with positive PD-L1 expression showing durable response to pembrolizumab. The role of pembrolizumab as monotherapy in advanced prostate cancer is being further investigated in the phase 2 KEYNOTE-199 study.

\section{Pembrolizumab added to enzalutamide}

This study was conceptualized on the observation that PD-L1 expression is increased following resistance to enzalutamide. ${ }^{6}$ Chemotherapy-naïve men with mCRPC progressing on enzalutamide were treated with four doses of pembrolizumab in this phase 2 study. Primary endpoint was PSA response rate, with secondary endpoints including ORR, PSA PFS, and OS.

To date, 28 patients have been enrolled. Interim data were presented at ESMO 2016. Three patients were still receiving the first four cycles of pembrolizumab at the time of presentation, while 25 had completed therapy. Of the 25, two received a second course of pembrolizumab.

Efficacy data were available for 27 patients, with a median followup of 19 weeks. Of these, five (18.5\%) had a confirmed PSA response and four of 19 patients had stable disease for greater than six months without a PSA response. None of the five responders have relapsed. Median PFS was 34 weeks. There have been seven Grade 3 or higher or immune-related AEs.

In summary, these early results indicate a clinically significant, and so far durable response to PD-1 inhibition in combination with continued enzalutamide treatment in men with $\mathrm{mCRPC}$.

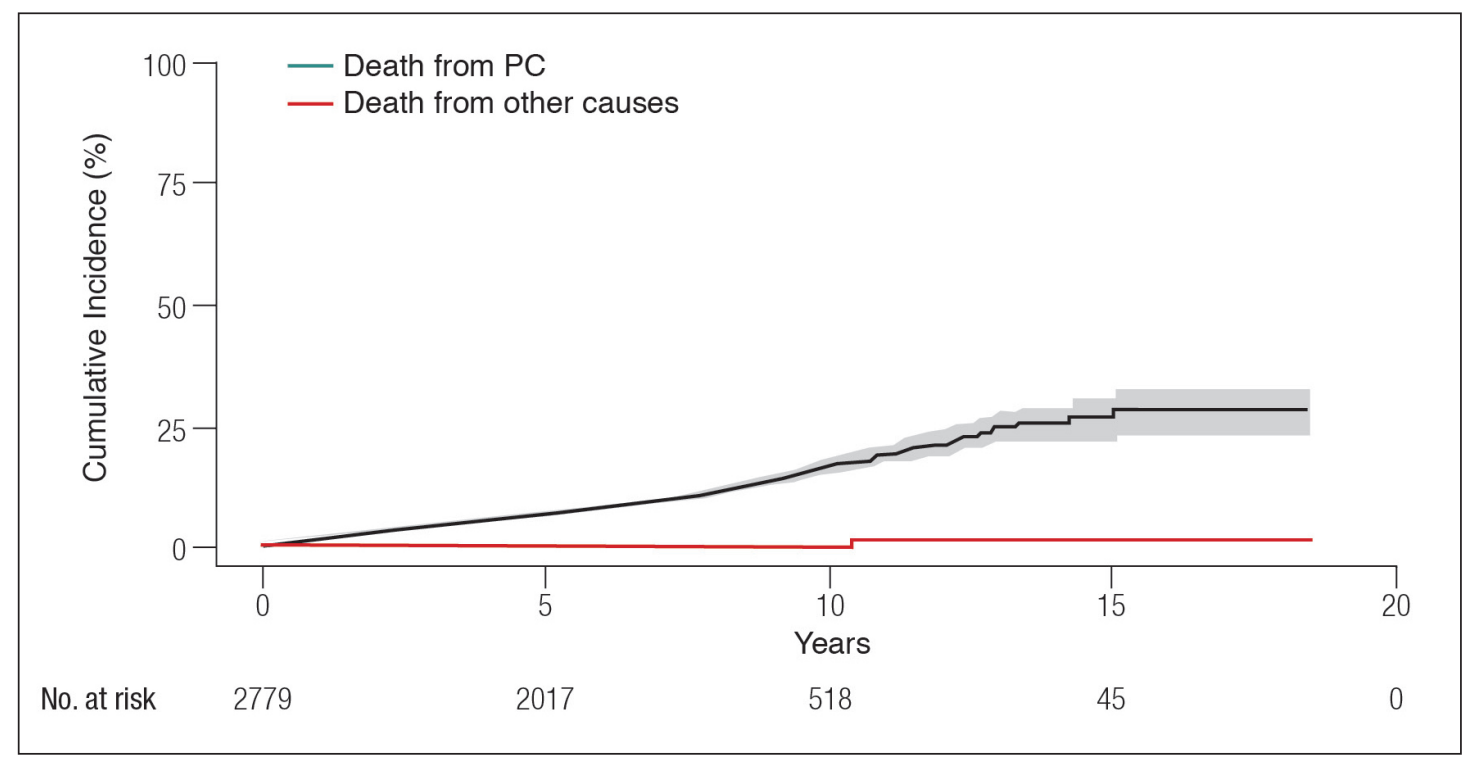

Fig. 2. Risk of dying from prostate cancer or other causes among men with initial benign transrectal ultrasound-guided biopsy set and prostate-specific antigen $\leq 10 \mathrm{mcg} / \mathrm{L}$. PC: prostate cancer. 


\section{Long-term followup of men with initial, benign needle core biopsy}

At ESMO 2016, Danish investigators presented the results of a long-term followup of men who had an initial benign transrectal ultrasound-guided biopsy (TRUS-gb) set. ${ }^{7}$ The principle aim of the study was to assess whether systematic biopsies indeed miss significant prostate cancers. The subjects included all Danish men with a benign TRUS-gb from 1995-2011 ( $n=27$ 537, median age 67 years, median PSA 7.7, median followup 7.1 years).

Out of this cohort of patients, $74.4 \%$ remained alive at the time of evaluation, 6695 had died, and 356 were lost to followup. For men with an initial benign biopsy, the cumulative risk of prostate cancer mortality and all-cause mortality was $5.2 \%$ and $59.9 \%$ after 20 years, respectively. When stratified by PSA at the time of biopsy, the estimated risk of prostate cancer mortality at 15 years in those with a benign biopsy and PSA of $<10 \mathrm{mcg} / \mathrm{L}$ was $0.7 \%$ (Fig. 2).

These results indicate that an initial benign prostate biopsy in the setting of low PSA predicts for a very low long-term risk of prostate cancer mortality. This data will help clinicians advise men on their long-term outlook when facing results of a prostate biopsy.

\section{References}

1. Sweeney C, Chen Y-H, Liu G, et al. Long-term efficacy and QOL data of chemohormonal therapy in low- and high-volume hormone-naïve metastatic prostate cancer: E3805 CHAARTED trial. Presented at ESMO 2016; Poster \#720PD.

2. Carles J, Davis ID, de Bono IS, et al. Safety of long-term treatment of chemotherapy-naïve metastatic castration-resistant prostate cancer patients with abiraterone acetate plus prednisone for $\geq 4$ years. Presented at ESMO 2016; Poster \#740P.

3. Grande E, Fernández-Perez MP, Font A, et al. Early responses to enzalutamide in AR-V7-positive first-line metastatic castration-resistant prostate cancer (mCRPC). A prospective SOGUG clinical trial: The PREMIERE study. Presented at ESMO 2016; Poster \#726PD.

4. Fizazi K, Hotte SJ, Saad F, et al. Final results from the AFFINITY phase 3 trial of custirsen and cabazitaxel/ prednisone in men with metastatic castration-resistant prostate cancer (mCRPC) previously treated with docetaxel. Presented at ESMO 2016; Presentation \#LBA9_PR.

5. Hansen A, Massard C, Ott PA, et al. Pembrolizumab for patients with advanced prostate adenocarcinoma: Preliminary results from the KEYNOTE-028 study. Presented at ESMO 2016; Poster \#725PD.

6. Graff JN, Alumkal JJ, Drake CG, et al. First evidence of significant clinical activity of PD-1 inhibitors in metastatic castration-resistant prostate cancer (mCRPC). Presented at ESMO 2016; Presentation \#7190.

7. Klemann N, Helgstrand JT, Brasso K, et al. Risk of prostate cancer mortality in men with an initial, benign needle core biopsy set: A population-based analysis with up to 20 years of followup. Presented at ESMO 2016; Poster \#724PD. 